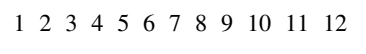




\title{
Data Exploitation of New Galileo Environmental Monitoring Units
}

\author{
I. Sandberg, S. Aminalragia-Giamini, G. Provatas, A. Hands, K. Ryden, D. Heynderickx, A. Tsigkanos, C. \\ Papadimitriou, T. Nagatsuma, H. Evans, and D. Rodgers
}

\begin{abstract}
The radiation environment of the Galileo spacecraft is severe and poorly characterized. The Galileo orbit takes the spacecraft through the heart of the outer radiation belt, while the low levels of geomagnetic shielding throughout the orbit expose the spacecraft to intermittent intense fluxes of protons during Solar Energetic Particle Events. In the Galileo constellation, two Environmental Monitoring Units (EMU) are currently flying in two different orbital planes. These units monitor the radiation environment and provide critical information related to hazards for the host spacecraft and its payload. In this work, we present results from the analysis of the surface charge collecting plates and of the proton telescope sensors. The performed numerical calibration of the EMU sensors and the application of novel unfolding and in-flight cross-calibration techniques allow the calculation of high quality proton and electron differential fluxes. The creation of a high-quality, long-term EMU electron flux dataset, is a step forward towards the improved characterization of MEO environment through the update of existing or the development of new radiation environment models.
\end{abstract}

Index Terms-Inverse problems, Electrons, Protons, Radiation monitoring, Semiconductor radiation detectors, Solar system, Space charge, Sun.

\section{INTRODUCTION}

$\mathbf{T}$ $\mathrm{HE}$ radiation environment in medium earth orbit (MEO) is rather severe for the satellites that spend therein a part or the whole duration of their mission. MEO orbit lies in the core of the dynamic outer electron radiation belt while it is

This work has been supported by ESA/ESTEC contract No. 4000119253/17/NL/LF/hh.

I. Sandberg is with the Space Applications \& Research Consultancy, 73 Aiolou St, 10551 Athens, Greece (corresponding author, phone: +302103257865; e-mail: sandberg@sparc.gr)

S. Aminalragia-Giamini is with the Space Applications \& Research Consultancy, 73 Aiolou St, 10551 Athens, Greece (sagiamini@sparc.gr)

G. Provatas is with the Space Applications \& Research Consultancy, 73 Aiolou St, 10551 Athens, Greece (gprovatas@sparc.gr)

A. Hands is with Surrey Space Centre, University of Surrey, United Kingdom, (a.hands@surrey.ac.uk)

K. Ryden is with Surrey Space Centre, University of Surrey, United Kingdom, (k.ryden@surrey.ac.uk)

D. Heynderickx is in DH Consultancy, Leuven, Belgium (dhconsultancy@skynet.be)

A. Tsigkanos is with the Space Applications \& Research Consultancy, 73 Aiolou St, 10551 Athens, Greece (antts@sparc.gr)

C. Papadimitriou is with the Space Applications \& Research Consultancy, 73 Aiolou St, 10551 Athens, Greece (constantinos@sparc.gr)

T. Nagatsuma is with National Institute of Information and Communications Technology,4-2-1 Nukui-kita, Koganei 184-8795, Tokyo, JAPAN (tnagatsu@nict.go.jp)

H. Evans is with ESTEC, European Space Agency, 2200 AG Noordwijk, The Netherlands (Hugh.Evans@esa.int)

D. Rodgers is with ESTEC, European Space Agency, 2200 AG Noordwijk, The Netherlands (David.Rodgers@esa.int) also subject to intense solar proton and heavy ion intermittent fluxes.

The intense electron fluxes of the outer radiation belt cause ionizing dose, non-ionizing energy loss (NIEL) and internal charging. Satellites in MEO are almost fully exposed to intermittent intense fluxes of protons due to the low levels of geomagnetic shielding. As a consequence, Solar Energetic Particle (SEP) events provide an additional contribution to dose and NIEL. In addition, heavy ions, originating from Galactic cosmic rays and SEP events can lead to single event effects on electronic components. Due to all these, spacecraft components are exposed to several types of radiation effects attributed to charged energetic particles.

For shielding and operations design, accurate electron environment models, based on reliable datasets, are needed in order to decrease the error margins and to anticipate in flight problems. Various modelling activities have been undertaken in recent years, using data from many satellite instruments to characterize the MEO electron environment. Data from an earlier version of the SURF instrument [1], which is still operating as part of the Merlin instrument suite on-board the test-bed satellite Galileo In-Orbit Validation Element A (GIOVE-A), has been used to create the Model of Outer Belt Electrons for Dielectric Internal Charging (MOBE-DIC) [2]. As the name suggests, this engineering model is targeted at a specific radiation effects concern, that of internal charging of spacecraft dielectrics. Office National d'Etudes et de Recherches Aérospatiales (ONERA) has used Los Alamos National Lab - Global Positioning System (LANL-GPS) data [3] to create a bespoke specification model for the constellation Galileo, which provides orbit-averaged spectra as a function of percentile [4]. This builds on the heritage of previous ONERA models developed for the MEO electron environment [5].

The largest and most significant effort in this area has been the development of a successor to the historic AE- 8 model. AE-9 [6] is a complex statistical model based on a large number of data sets. AE-9 can be run in different modes, in terms of how statistical fluctuations due to instrumental uncertainties and space weather, are treated. Other models exist too, of course. However, AE-9 is likely to become the industry standard and any new data sets are thus likely to be compared with its output in order to validate instrument calibration against a range of model flux predictions.

During the last decades, European radiation monitor units, such as Merlin (see [7] and references therein) and ESA Standard Radiation Environment Monitor (SREM) [8] on board GIOVE-A and -B missions have provided measurements 


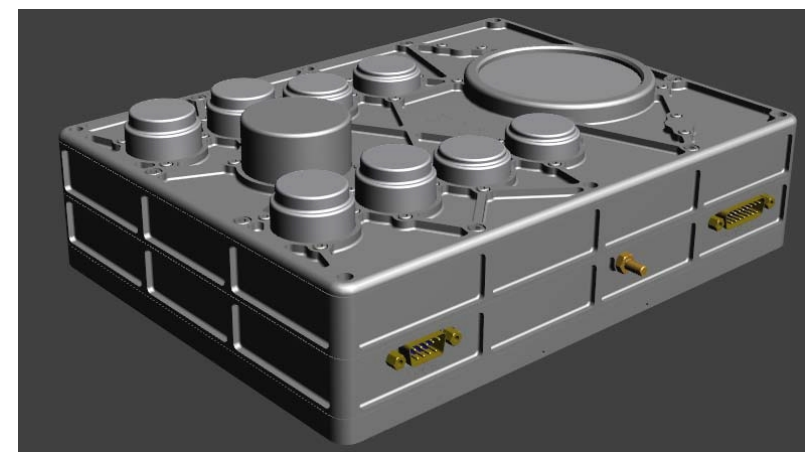

Fig. 1. The EMU unit on-board GSAT0207 and GSAT0215

of great interest for MEO environment. In the Galileo constellation, two radiation monitors are currently flying in two different orbital planes. The first Environmental Monitoring Unit (EMU) is on-board Galileo satellite (GSAT) 0207 (also called Antonianna) which was launched on 17 November 2016, while a second EMU is on board GSAT0215 (also called Nicole) which was launched on 12 December 2017. Remarkably, two additional units of a virtually identical instrument, the Space Environment Data Acquisition Monitor (SEDA), are on-board two Japanese Meteorological Agency (JMA) geostationary (GEO) satellites. The SEDA units on-board Himawari-8 (launched on 7 October 2014) and Himawari-9 (launched on 2 November 2016) provide useful measurements for the characterization of GEO environment during the last years [9].

In this work, we present new calibration approaches and selected results from the analysis of the data collected by the charge collecting plates (SURF sensor) and of the proton telescope (PT) sensors of EMU. The methods developed were tested and applied to both GSAT0207/EMU and Himawari8/SEDA datasets.

In Sec. II, we summarize the main characteristics of the EMU, while in Sec. III we highlight the performed numerical calibrations and the unfolding techniques applied for the derivation of electron and proton differential fluxes. In Sec. IV we present detailed results on the analysis of EMU/SURF data and electron fluxes using the derived response function and we introduce a novel method for the in-flight cross-calibration of EMU/SURF. In Sec. V, we present results on the analysis of EMU/PT measurements and the derived proton fluxes, and perform cross-calibration studies. Last, in Sec. VI] we present the main conclusions of the conducted work and the items to be addressed in the future.

\section{The EnVIRONMENTAL MONitoring Unit}

The Environmental Monitoring Unit (EMU) is a radiation sensitive instrument designed for use in the Galileo orbit. Its design is based on the heritage of the SURF, the Cosmic Radiation Environment and Dosimetry (CREDO) instrument and Merlin (see [7] and references therein) developed by UK teams. It is manufactured by Thales-Alenia Space, Switzerland.

EMU comprises four different types of sensors:
- SURF: a stack of charge collecting plates which measures internal charging currents under shielding, itself of importance for assessing the space weather threat but from which differential electron fluxes can also be unfolded. The eight plates in the stack provide a wider and better resolved energy response than previously obtained from GIOVE-A/Merlin.

- A set of eight proton telescopes (PT) which measure energetic proton fluxes. The eight separate detectors have different shielding levels to provide energy discrimination.

- A heavy ion sensor to measure Linear Energy Transfer (LET) spectra from Galactic Cosmic Rays and SEP events. This is the same dual diode telescope as used in Merlin.

- RadFETS which measure internal doses inside the instrument. These are distributed inside the instrument.

\section{Numerical CALIBRATIONS OF EMU SENSORS}

The voltage measurements of SURF plates $V_{i}, i=1 . .8$ are given by the following relation,

$$
V_{i}=V_{o f f, i}+C_{V} \int_{0}^{\infty} f_{e}(E) R F_{i, e}(E) d E
$$

and are attributed to the incident electron flux $f_{e}(E)$ $\left[\mathrm{cm}^{-2} \mathrm{MeV}^{-1} \mathrm{~s}^{-1} \mathrm{str}^{-1}\right]$. Here, $R F_{i, e}(E)[A \mathrm{str}]$ denotes the response of the SURF plates, $V_{o f f, i}$ a characteristic voltage offset, and $C_{V}\left[\mathrm{~cm}^{2} \Omega\right]$ is a constant which depends on the channel amplifier characteristics. Similarly, the measured Proton Telescopes count-rates $C_{i}, i=1 . .8$ are given by:

$$
C_{i}=\int_{0}^{\infty} f_{p}(E) R F_{i, p}(E) d E
$$

and are attributed to the incident proton flux $f_{p}(E)$ $\left[\mathrm{cm}^{-2} \mathrm{MeV}^{-1} \mathrm{~s}^{-1} \mathrm{str}^{-1}\right]$ (here $R F_{i, p}(E)\left[\mathrm{cm}^{2} s t r\right]$ ).

The SURF charging sensor and the 8 proton-telescopes have been calibrated to mono-energetic electrons and protons by means of Monte-Carlo Geant4 [10] simulations. The latter were configured with Geant 4 v.9.6 patch-03 and were carried out using Geant4 Radiation Analysis for Space (GRAS v.03-03-r1561) [11] platform. The Option 3 of the default electromagnetic (EM) constructor was used, designed for any applications required higher accuracy of electrons, hadrons and ion tracking without magnetic field. The geometry of the instrument was constructed by means of the ROOT v.5.34/20 [12] geometry package and exported to GDML format. The model that was imported in the GRAS/Geant4 simulations was based on the configuration of the instrument. In this model all the sensors of EMU were included as well as the various other shields, PCB boards etc. At the bottom of the EMU a simplified satellite model, consisting of a $3 \mathrm{~mm}$ thick $\mathrm{Al}$ sheet, was also included in the geometry file.

In the upper plot of Figure 2, the absolute values of the obtained electron responses for SURF are presented. The responses present approximately the same maximum values for the seven out of eight different SURF plates. In the lower plot of Figure 2 the proton response functions of the proton 

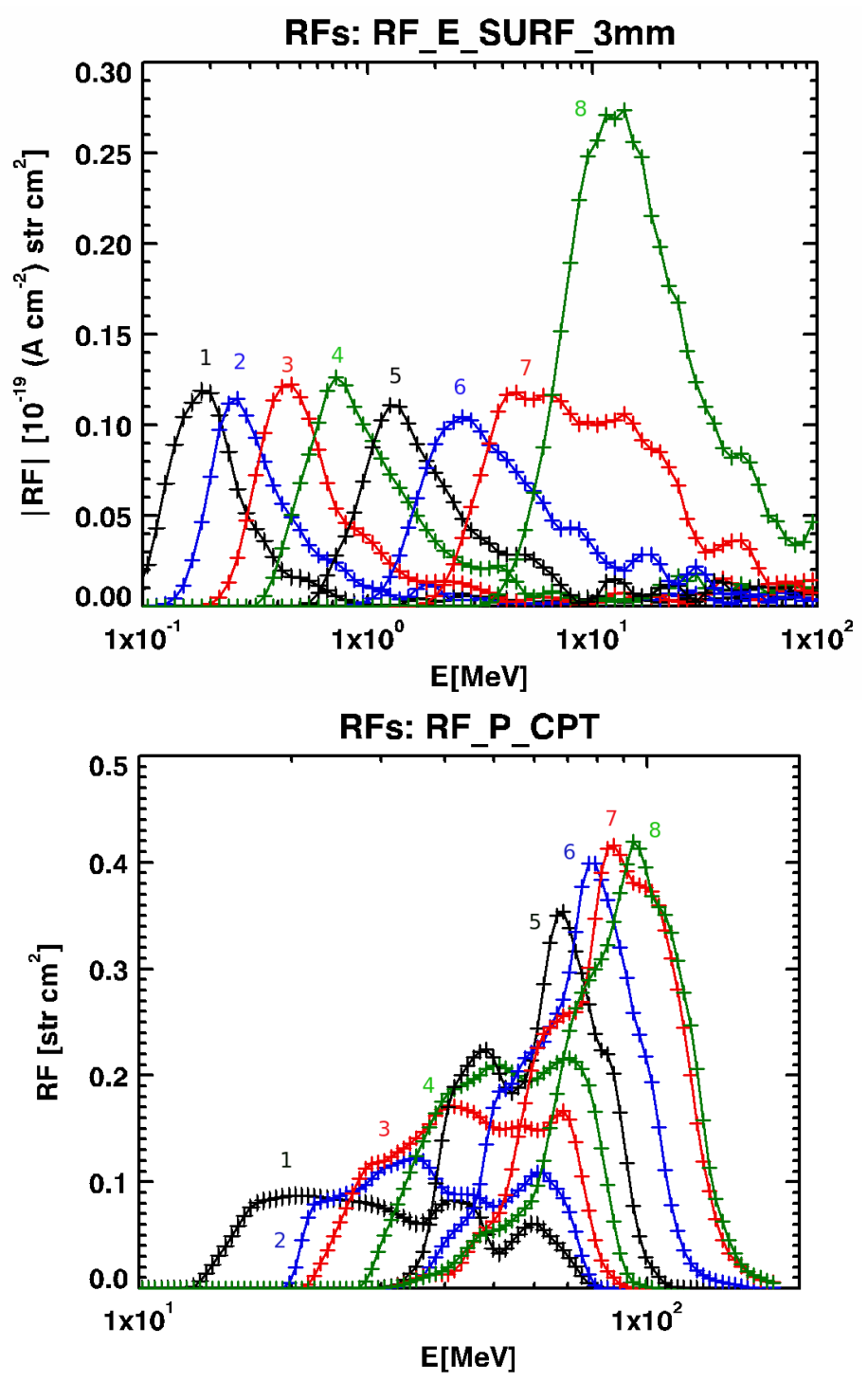

Fig. 2. The electron response functions of EMU/SURF plates (upper plot) and the proton response functions for EMU proton telescopes (lower plot) when operating in coincidence mode.

telescopes operating in coincidence mode are presented. There is an evident trend that the maximum response of the PTs gets higher as the peak energy increases. This is a result of the different shielding and energy-threshold settings of the sensors. It should be mentioned here that the coincidence channels have lower responses, down to a factor of 100 , compared to the corresponding non-coincidence channel (not shown here).

The obtained response functions are used in the application of unfolding methods in order to derive the corresponding particle fluxes. The main pre-processing of the raw EMU data - required prior to the calculation of the differential fluxes - concerned the evaluation and subtraction of the time- and temperature- dependant offsets in the voltage measurements of SURF plates. In addition, pre-cautions were taken to use SURF low sensitivity data for the relatively limited cases when the high sensitivity SURF measurements exhibited saturation.
For given measurements of EMU, the calculation of the electron (proton) differential fluxes requires the inversion of Eq. 11 (or Eq. 2). This equation is a Fredholm integral equation of the first kind and is a classical example of an ill-posed problem as its solution is not unique. For the calculation of EMU proton and electron fluxes, we applied two unfolding techniques. The bow-tie (BT) analysis and the genetic correlative unfolding method (GenCORUM). These methods permit the calculation of the fluxes, for given response functions. The development of these methods for EMU was initially based on the analysis of Himawari-8/SEDA data - using the response functions presented in Sec. III However, in this work we focus on the analysis of GSAT0207/EMU measurements.

The BT analysis (see e.g. [13]) uses a representative family of charged particle spectra to estimate a characteristic energy at which the spread of the linear conversion factors - due to natural variability of the spectra indices - is minimized. The success of its applicability depends on the profiles of the response function in combination with the measured radiation environment. The BT analysis - when applicable - allows the derivation of a single factor which allows the direct multiplicative conversion of a channel measurement to a differential (or integral) flux value at the BT energy. The GenCORUM method [14] is a novel method based on a correlative comparison between actual and a virtual set of measurements. The latter ones are created by folding the response functions with analytical functions modeling particle flux spectra. Each measured count-rate is correlated with the set of pre-calculated virtual measurements. The best match that of highest correlation - is found and a scaling factor is derived and applied to the virtual flux from which the virtual count-rate was calculated. Following this, a genetic algorithm is further employed to fine-tune the flux spectra and optimize the agreement with the measurements.

The GenCORUM method is more general and permits the derivation of reliable particle flux spectra where the BT analysis fails. For the case of EMU, the use of GenCORUM allowed us to cross-validate the BT analysis. In what follows, we highlight on the results derived through the application of the BT method.

\section{ANALYSIS OF EMU/SURF MEASUREMENTS}

For the BT analysis of the response of SURF plates, we considered independently power-law and exponential spectra. The distribution of the considered spectral indices was calculated by performing spectral fits in the energetic electron flux dataset of Van Allen Probes (VAP) - formerly known as the Radiation Belt Storm Probes (RBSP).

In Figure 3, we present as an example of the BT analysis the results accounting for the electron response of the third EMU/SURF plate assuming exponential spectra. The multicolored curves map the conversion factors one should apply to convert data to differential fluxes for different energies. Each colored curve corresponds to different spectral index $1 / E_{0}$ (selected values of $E_{0}$ appear in the legend at the top left part of the plot). The dashed line - perpendicular to the $\mathrm{x}$-axis - depicts the characteristic BT energy, while the dashed lines - parallel 


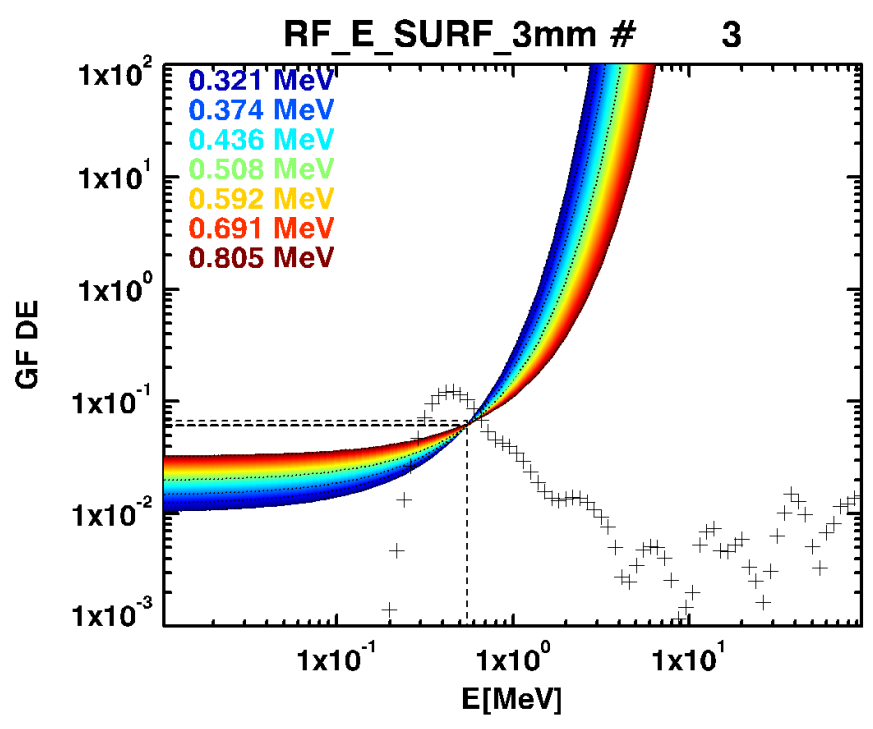

Fig. 3. Bow-tie analysis results for the third plate of SURF considerin exponential functions for the modelling of the electron flux spectra (the $y$ axis has arbitrary units). The black crosses denote the response function.

to the x-axis - depict the corresponding scaling factors of the quantiles of the sampled indices for given energy. Last, the black crosses represent the corresponding electron responst function. The bow-tie energy value is defined as the one which minimize the spread in the scaling factors accounting for the distribution of the measured spectral indices.

An overview of the BT results of the analysis of SURF responses is presented in Figure 4. The derived BT energies and scaling factors differ as they depend on the assumptions (i.e. power- or exponential law) for the spectral shape. I should be also noticed that the spread of the derived scalins factors for the different quantiles of the sampled spectral index distribution is relatively larger for the case of exponential spectra.

Table I presents the electron BT energies for the eight EMU/SURF plates, calculated assuming power-law (second column) and exponential spectra (third column). Using these energies and the median values of the distribution of the BT scaling factors, electron flux datasets can be constructed using SURF voltage measurements. In order to evaluate the differences between the exponential and the power-law derived BT EMU electron fluxes, we re-bined the BT-PL flux dataset to BT-EXP energies and compared the resulted flux series. The flux differences were evaluated by different means, i.e. by using RMS or $\chi^{2}$ values, and found to be insignificant for $E<2 \mathrm{MeV}$.

In what follows, we present and analyze results of the BT SURF electron flux series constructed by considering the exponential BT results for SURF plates 3-6 and the powerlaw BT results for plates 1-2 and 7-8. This combination was selected as it was found that optimize quantitatively the agreement between actual and reconstructed measurements, where the latter were calculated using Eq. 1. For the evaluation and the cross-calibration of the EMU SURF measurements and resulted fluxes, we made a series of comparisons using
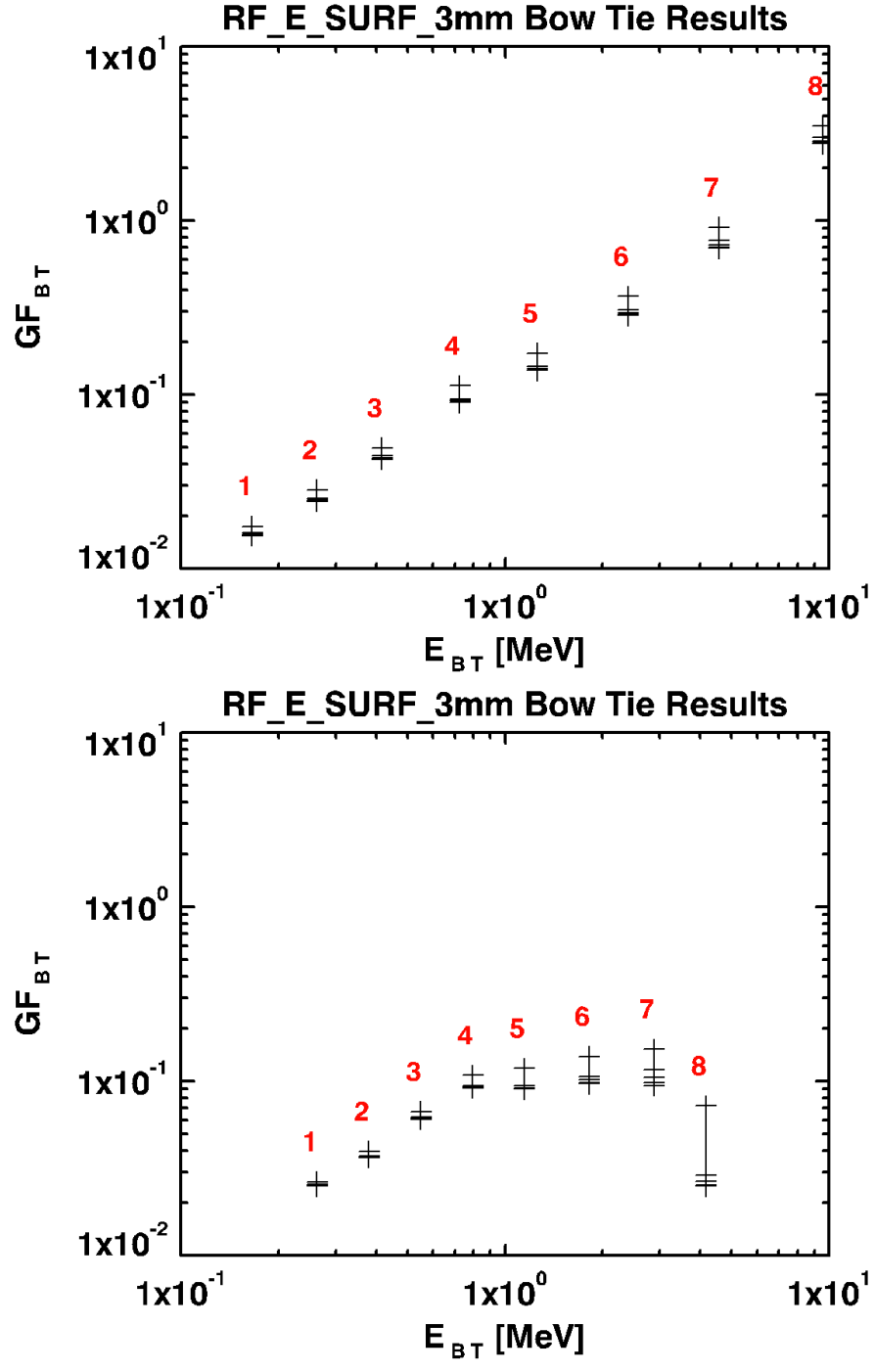

Fig. 4. Summary results of the Bow-Tie analysis of EMU/SURF electron responses. The geometric factors - in arbitrary units - versus the BT energy assuming power-law (upper panel) and exponential spectra (lower panel) for the electron fluxes.

science-level measurements from VAP-A [16] including both MagEIS [17] (level 2, release 4) and REPT [18] (level 2, release 3) data. The nominal electron energy range of MagEIS channels is within $0.03-4.062 \mathrm{MeV}$, while that of REPT is within 1.9-12.3 MeV. It should be noticed, that within the overlapping energy range, i.e. 1.9-4 $\mathrm{MeV}$ the flux measurements of MagEIS and REPT present differences which are reflected not only to the measurements per se but in the spectral index within that energy. Moreover, the highest energy channels of MagEIS seems to present enhanced background levels. In the studies that follow, we consider as VAP reference electron flux dataset, the collection of MagEIS measurements till $E=1.72$ $\mathrm{MeV}$ and REPT data within $E=1.9-7.7 \mathrm{MeV}$.

\section{A. Evaluation of EMU/SURF data products.}

For the evaluation of EMU/SURF responses and data products, we first determined suitable conjunctions between the target GSAT0207 and the reference (VAP-A) satellites. The 
TABLE I

CHARACTERISTIC ENERGIES OF SURF PLATES DERIVED USING BOW-TIE AND IN-FLIGHT CROSS-CALIBRATION ANALYSIS.

\begin{tabular}{|c||c|c|c|}
\hline SURF & $E_{B T, P L}[\mathrm{MeV}]$ & $E_{B T, E X P}[\mathrm{MeV}]$ & $E_{\text {eff }}[\mathrm{MeV}]$ \\
\hline 1 & 0.17 & 0.26 & 0.23 \\
2 & 0.26 & 0.38 & 0.31 \\
3 & 0.41 & 0.55 & 0.52 \\
4 & 0.72 & 0.79 & 0.81 \\
5 & 1.26 & 1.14 & 1.17 \\
6 & 2.40 & 1.82 & 1.76 \\
7 & 4.57 & 2.88 & 3.96 \\
8 & 9.54 & 4.16 & 6.07 \\
\hline
\end{tabular}

conjunctions are defined here as the spatiotemporal locations where the respective instruments expect to measure the same trapped electron environment - according to the dynamics of the charged particles inside Earth magnetosphere. The criteria for obtaining these positions are usually defined on the basis of adiabatic invariants. We adopted the concept of the recommendations of the Panel on Radiation Belt Environment Modeling (PRBEM/COSPAR) [20] but applied even stricter conditions:

- $4.6 \leq L \leq 4.9$,

- $1 \leq\left(B / B_{e q}\right) \leq 1.02$,

- $\delta t=2$ hours,

- $\delta(M L T)=2$ hours,

- $\delta L=0.01$, and

- $\delta\left(B / B_{e q}\right)=0.01$.

The first constraint requires particle measurements to be on closed magneto-shells. The magnetic field limitations ensure that both instruments may count the same distribution of particles bouncing along a magnetic line close to the geomagnetic equator. For the application of the conjunction criteria defined above, we calculated the magnetic coordinates variables using the UNILIB library [19] and we found 194 multiple magnetic conjunctions of GSAT0207 with VAP-A. The term multiple refers to the situation where a given instance of GSAT0207 measurements satisfy the conjunction criteria with an orbital segment of VAP-A that contain a continuous series of electron flux measurements. The appearance of multiple conjunctions is attributed to the one order of magnitude difference between the time-resolution of the target and the reference datasets.

In what follows, we present comparisons of the derived BT EMU omni-directional differential electron flux (FEDO) products versus the VAP-A spin-averaged differential electron fluxes (FESA). For each multiple conjunction, we have averaged the VAP-A fluxes over each orbital segment that satisfied the conjunction criteria with EMU. In Figure 5 we present - for brevity - results for five SURF plates. The plots in the left-hand-side panel of the figure present comparisons of SURF measurements versus reconstructed datasets. The reconstructed datasets have been calculated by integrating either the BT-derived EMU/FEDO spectra (blue crosses) or the VAP-A/FESA spectra (red crosses) along the electron energy range of SURF response functions. The plots in the righthand-side panel of the figure, present direct comparisons of the BT-derived EMU/FEDO fluxes with the reference VAPA/FESA averaged measurements during the conjunctions.

Table II presents some quantitative characteristics of the
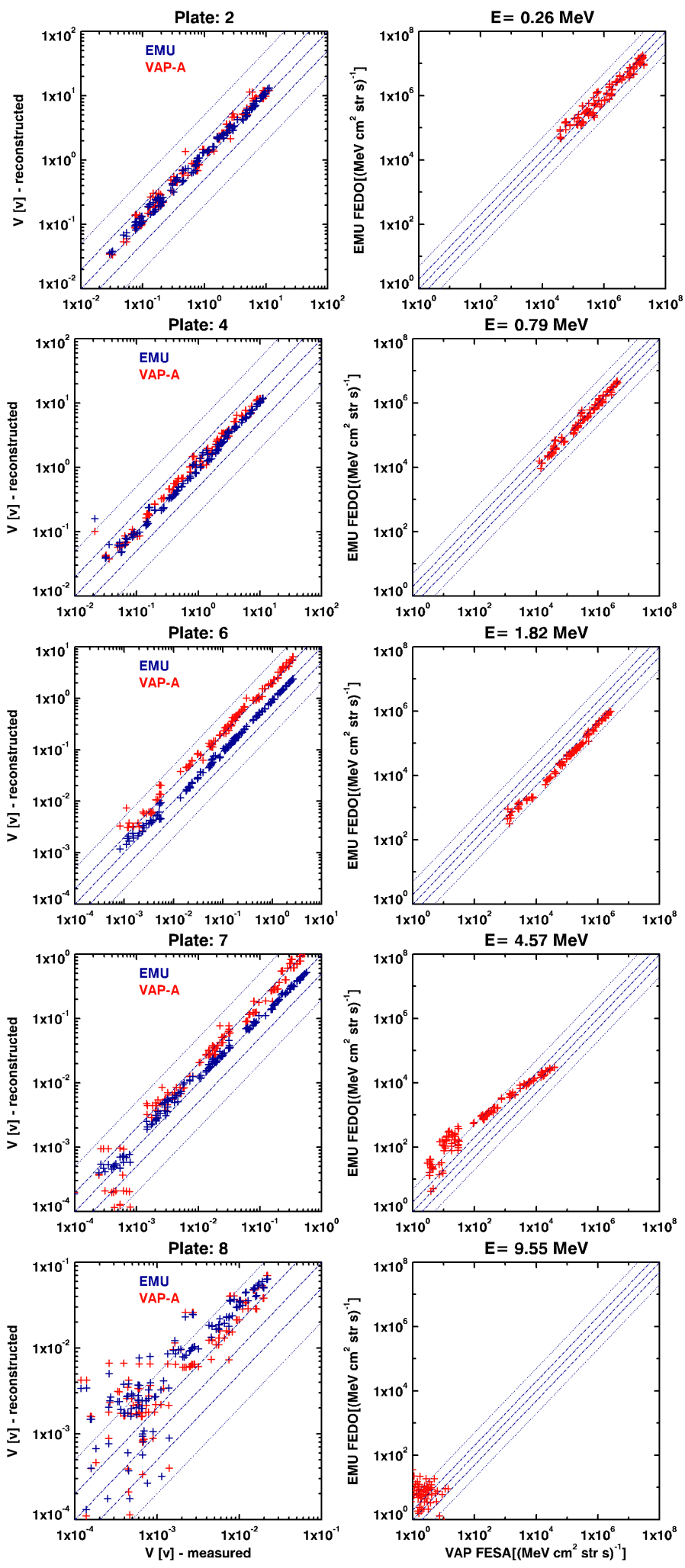

Fig. 5. Comparisons of BT EMU/FEDO fluxes during suitable conjunctions. The cross-plots on the left-hand-side present comparisons of reconstructed versus actual EMU/SURF voltage measurements, calculated by folding the BT EMU/FEDO (blue crosses) and the reference VAP-A/FESA (red crosses) fluxes with SURF response functions. The plots on the right-hand-side present comparisons between the BT EMU/FEDO and the reference VAP-A FESA fluxes re-binned at the BT energies. 
TABLE II

QUARTILES OF THE RATIOS OF ELECTRON DIFFERENTIAL FLUXES CALCULATED FROM VAP-A ENERGETIC ELECTRON DETECTORS AND FROM EMU/SURF

\begin{tabular}{|c|c||c|c|c|}
\hline Detector & $E_{B T}[\mathrm{MeV}]$ & $Q_{1}$ & Median $Q_{2}$ & $Q_{3}$ \\
\hline MagEIS & 0.17 & 0.67 & 1.15 & 1.87 \\
& 0.26 & 0.82 & 1.03 & 1.21 \\
& 0.55 & 0.78 & 0.87 & 0.98 \\
& 0.79 & 0.91 & 1.06 & 1.17 \\
& 1.16 & 1.05 & 1.15 & 1.29 \\
& 1.82 & 1.73 & 1.92 & 2.15 \\
& 4.57 & 2.97 & 3.94 & 11.6 \\
\hline REPT & 1.82 & 6.45 & 7.61 & 8.57 \\
& 4.57 & 0.18 & 0.27 & 0.59 \\
\hline
\end{tabular}

comparisons between EMU/FEDO fluxes and VAP-A fluxes. We have used as a metric for comparisons the quartiles of the ratios of VAP-A electron differential fluxes (re-binned at $\mathrm{BT}$ energies) to the EMU BT fluxes. In order to highlight the inherent differences in the reference datasets, we have selected to present comparisons with MagEIS and REPT datasets separately.

The quartiles in Table II indicate that the agreement between EMU and VAP-A/MagEIS fluxes is remarkable for energies below $1.82 \mathrm{MeV}$. This validates the performance of the EMU/SURF sensor, the performed numerical calibrations of EMU/SURF (with respect to MagEIS calibration) and the success of the BT analysis for - at least - the first five plates. For $1.82 \mathrm{MeV}$ the ratios are of the order of two (for MagEIS) to 7 (for REPT). An inspection in the comparison plot between the actual and the reconstructed measurements of the corresponding plate (Plate 6, left plot of Figure 5) indicates, however, that the reference (MagEIS) measurements at this energy are not consistent with SURF measurements; as a consequence the differences of the flux products are not attributed to the unfolding procedure.

The differences at highest energies are more dramatic and may be attributed to the synergy of various reasons; the accuracy of SURF response functions due to the unspecified shielding attributed to spacecraft body, the applicability of the BT approach and to the calibration of REPT, which present consistent differences with respect to MAGEIS. The latter is also highlighted by the differences in the median values for $4.57 \mathrm{MeV}$.

\section{B. In flight calibration of EMU/SURF electron fluxes.}

For the further evaluation and calibration of EMU SURF measurements, we have developed and applied a novel method. The method - which will be referred here as the effective energy calibration method - permits the calculation of EMU/SURF electron fluxes without using any a priori information of the SURF response functions. The method is based on the determination of the most characteristic energy values of SURF plates, with the use of the reference flux dataset, and the subsequent calculation of EMU/SURF electron differential fluxes.

The method is a generalization of the approach developed by Sandberg et al. [22] for the cross-calibration of solar proton flux datasets of NOAA GOES units using NASA IMP-8/GME dataset. The effective energy in Ref. [22] was defined as the value one should attribute to the differential flux series of the target dataset (i.e. GOES/EPS) to optimize the agreement with the reference dataset (i.e. IMP-8/GME). In the present work, the effective energy $E_{\text {eff }}$ is defined as the energy one should choose to re-bin (i.e. perform a linear piecewise interpolation in the log-space) using the reference dataset (i.e. VAP-A) in order to optimize the cross-calibration of SURF and the subsequent calculation of the differential flux series. The application of the method follows the steps:

- Re-bin the flux series of the reference dataset (i.e. VAPA) into a dense energy grid.

- For the determined conjunctions, calculate the $\chi^{2}(E)$ values between the logarithmic values of the target instrument measurements (SURF voltages) and the reference (VAP-A/FESA) flux series.

- Define as $E_{\text {eff }}$ the energy that minimizes $\chi^{2}(E)$.

- Calibrate SURF measurements with VAP-A/FESA using the linear fit results for $E=E_{\text {eff }}$ and derive EMU/FEDO.

The effective energies, derived with the in-flight crosscalibration method are presented in the third column of Table I. The majority of the effective values are in close agreement with the energy values derived by assuming exponential spectra in the BT analysis (third column in Table I). It should be underlined here that the presented cross-calibration method when applied to the FEDO BT fluxes - instead of the $V_{S U R F}$ measurements - leads exactly to the same results. This is attributed to the fact that the BT fluxes correspond to the rescaling of voltage SURF measurements and the method for the determination of the effective energy, which is based on the $\chi^{2}(E)$-values of logarithms.

In Figure 6, we present comparisons of the derived effective EMU omni-directional differential electron flux products versus the VAP-A spin-averaged differential electron fluxes. The structure and the description of the plots in the figure is similar to those for Figure 5. It is evident that using the effective calibration method, EMU fluxes up to $6 \mathrm{MeV}$ can be derived that present excellent agreement with VAP-A reference flux dataset. However, as it can be also seen the reconstructed measurements are not in agreement with the actual ones.

Last, a comparison of the mean FEDO fluxes derived using the BT, the GenCORUM and the effective cross calibration methods is presented in Figure 7 The mutual agreement of the BT and the GenCORUM EMU FEDO spectra, in the energy range up to $1 \mathrm{MeV}$, with the VAP-A/MagEIS dataset is remarkable. In the energy range of about $E=1-2 \mathrm{MeV}$, the $\mathrm{BT}$ and the GenCORUM EMU FEDO spectra preserve their agreement, while deviations with VAP-A fluxes start to appear. It should be recalled here that this is the buffer zone between MAGEIS rel. 4 and REPT rel. 3 datasets where mismatches in the merged spectra are expected due to the different characteristics and calibration of the instruments. Obviously, the effective mean EMU/FEDO spectra is in absolute agreement with VAP-A.

\section{ANALYSIS OF EMU/PT MEASUREMENTS}

For the case of EMU proton telescopes, we applied a BT 

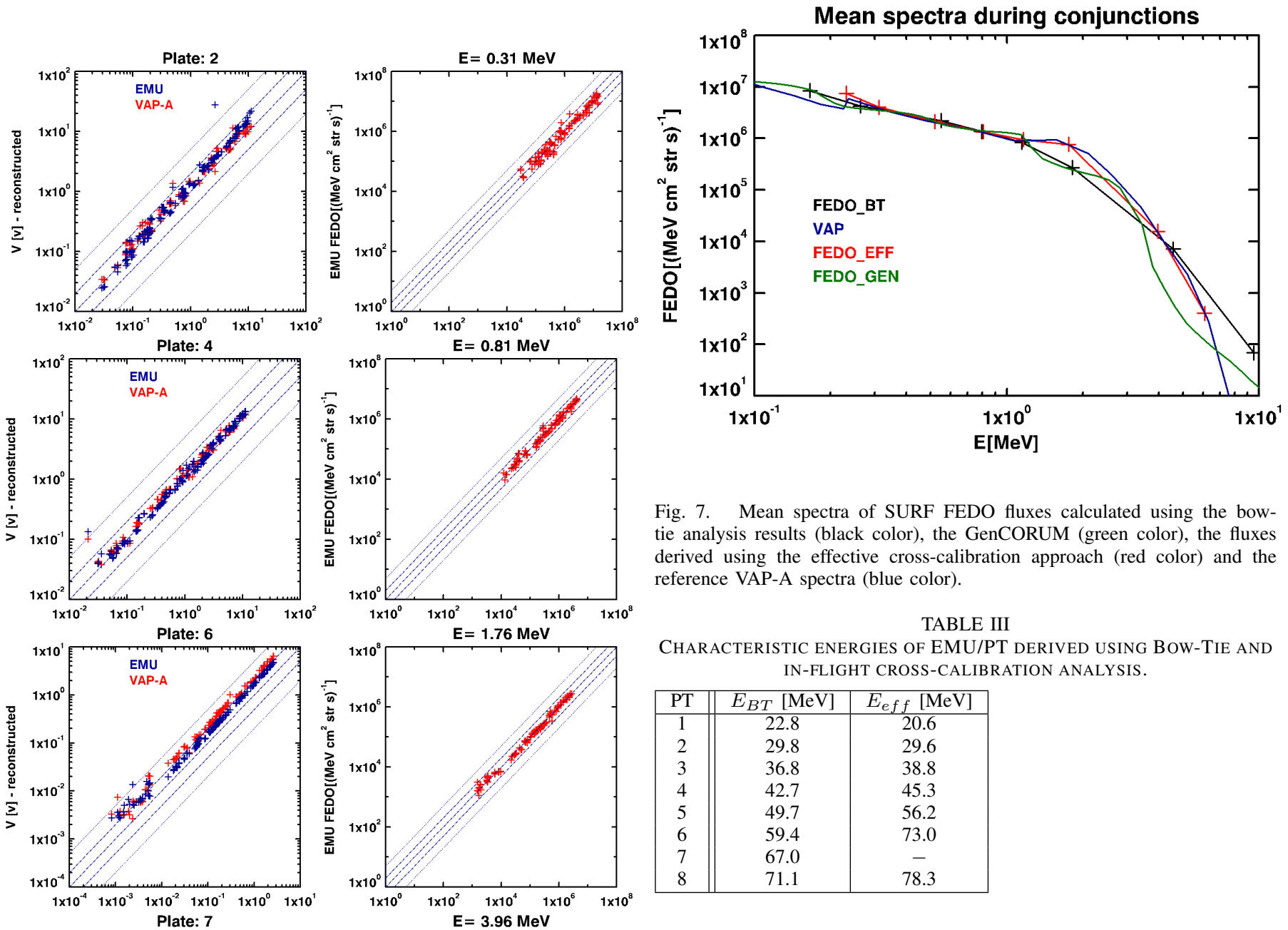

Fig. 7. Mean spectra of SURF FEDO fluxes calculated using the bowtie analysis results (black color), the GenCORUM (green color), the fluxes derived using the effective cross-calibration approach (red color) and the reference VAP-A spectra (blue color).

\section{TABLE III}

CHARACTERISTIC ENERGIES OF EMU/PT DERIVED USING BOW-TIE AND IN-FLIGHT CROSS-CALIBRATION ANALYSIS.

\begin{tabular}{|c||c|c|}
\hline PT & $E_{B T}[\mathrm{MeV}]$ & $E_{\text {eff }}[\mathrm{MeV}]$ \\
\hline 1 & 22.8 & 20.6 \\
2 & 29.8 & 29.6 \\
3 & 36.8 & 38.8 \\
4 & 42.7 & 45.3 \\
5 & 49.7 & 56.2 \\
6 & 59.4 & 73.0 \\
7 & 67.0 & - \\
8 & 71.1 & 78.3 \\
\hline
\end{tabular}
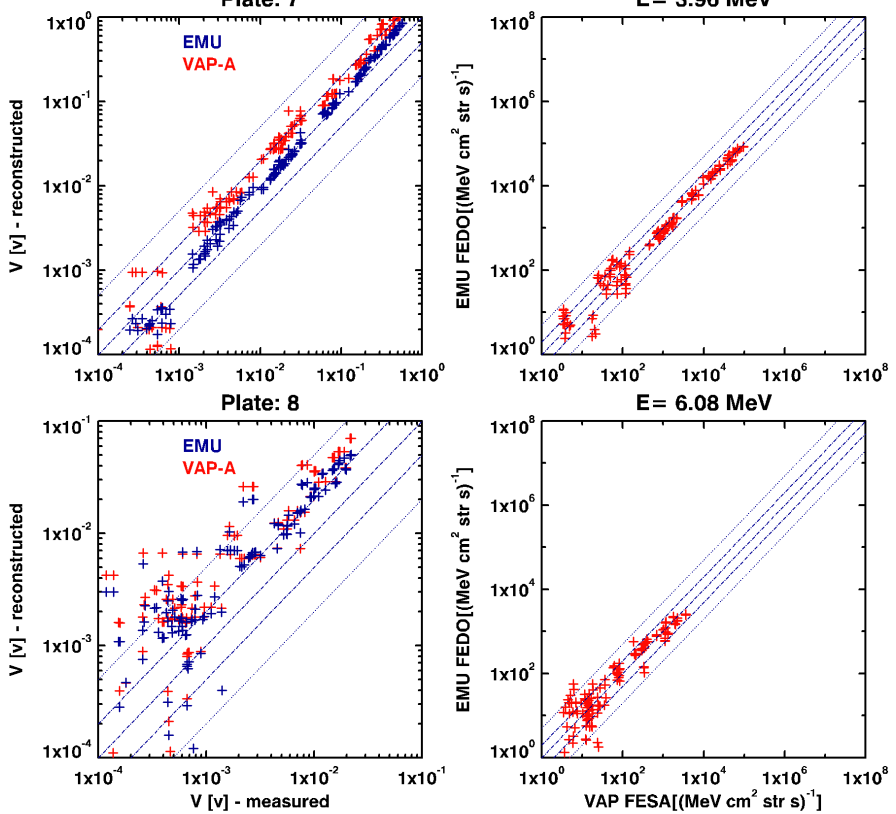

Fig. 6. Comparisons of effective EMU/FEDO and VAP-A fluxes during the selected conjunctions. The cross-plots on the left-hand-side present comparisons of reconstructed versus actual EMU/SURF voltage measurements, calculated by folding the effective EMU/FEDO (blue crosses) and the reference VAP-A/FESA (red crosses) fluxes with SURF response functions. The plots on the right-hand-side present comparisons between the effective EMU/FEDO and the reference VAP-A FESA fluxes re-binned at the effective energies.

analysis assuming power-law spectra. The distribution of the considered spectral indices was sampled from ESA SEPEM (Solar Energetic Particle Environment Modelling) Reference Dataset 2.0 (RDS) [15], which contain proton flux data from 1974 , within the energy range of $10-200 \mathrm{MeV}$. The RDS contains processed data from the NOAA Energetic Particles Sensor (EPS), part of the Space Environment Monitor (SEM) package on-board GOES and earlier SMS (Synchronous Metereological satellites). The proton flux data have been cleaned and cross-calibrated to find their effective energy [22] using measurements from the Goddard Medium Energy (GME) instrument on-board NASA IMP-8 spacecraft. The derived bow-tie energy values for the EMU/PT are presented in the first column of Table III.

The performed comparisons are limited to the proton flux enhancements during the SPEs of September 2017 [21]. The following steps were employed:

- The proton fluxes of EMU/PT were derived using the results of the BT analysis.

- Measurements of EMU/PT for non-defined $L$ values were retained in order to exclude regions of magnetospheric shielding and regions of trapped electron radiation.

- The RDS data were interpolated at the times of EMU/PT measurements.

- (New) effective energies to characterize the BT-derived 


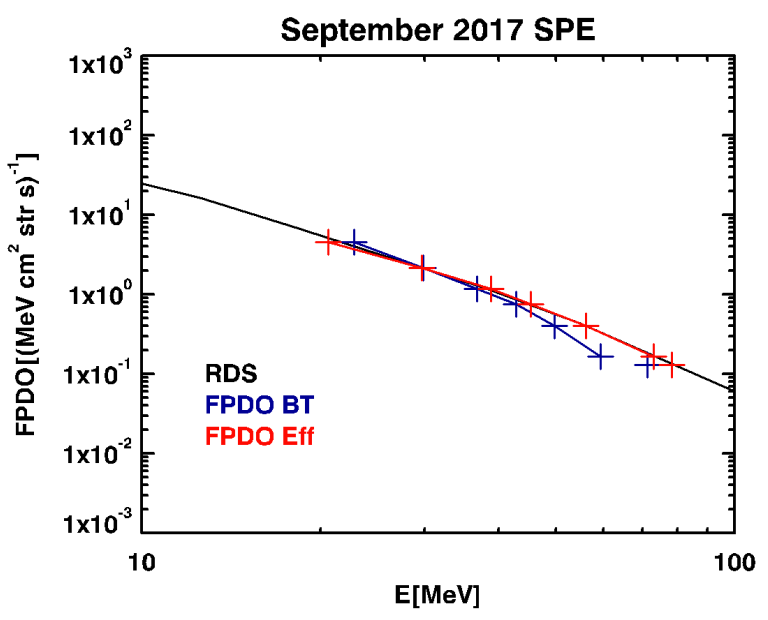

Fig. 8. Mean spectra of the BT EMU proton flux series for the September 2017 SPEs, using the BT (blue color) and the effective energies (red color) versus the SEPEM RDS spectra (black curve).

proton flux series were determined using the crosscalibration method described in Ref. [22].

The effective energy method updates the energy values of the derived flux series leaving untouched the proton flux series. The derived effective energies are presented in the second column of Table III. As one may see, the values are - in most of the cases - in excellent agreement with the BT energies. A comparison of the mean BT FPDO flux spectra, using the BT and the effective energies versus the RDS data is presented in Figure 8

In addition, in Figure 9, we present detailed comparisons of the BT EMU proton flux series versus the RDS dataset re-binned at the effective energies. The plots in the left-handside panel present comparisons of the proton flux profiles during the SPEs - while the plots in the right-hand-side are just the corresponding cross-plots. It is evident that the use of the effective energies serve as a direct cross-calibration method for the derived BT EMU proton fluxes. It should be pointed out here, that the background levels of the EMU/PT fluxes are smaller than those of the RDS.

\section{Vi. Conclusions}

In this work, we present new results emerged from the analysis of EMU data on-board GSAT0207. We have calculated by means of GEANT4 simulations the response functions of the proton and electron sensors of EMU and applied dedicated numerical techniques for the calculation and the in-flight cross-calibration of electron and proton fluxes. The developed methods were initially applied on Himawari-8/SEDA data. The flux products of GSAT0207/EMU were compared, evaluated and cross-calibrated successfully with VAP-A (for the case of SURF sensor) and GOES/EPS data (for the case of PT sensors). The analysis of the measurements of the SURF and PT sensors of EMU will allow the construction of high-quality electron and proton flux datasets.

The derivation of GSAT0207/EMU and GSAT0215/EMU electron fluxes will provide to the community new and reliable
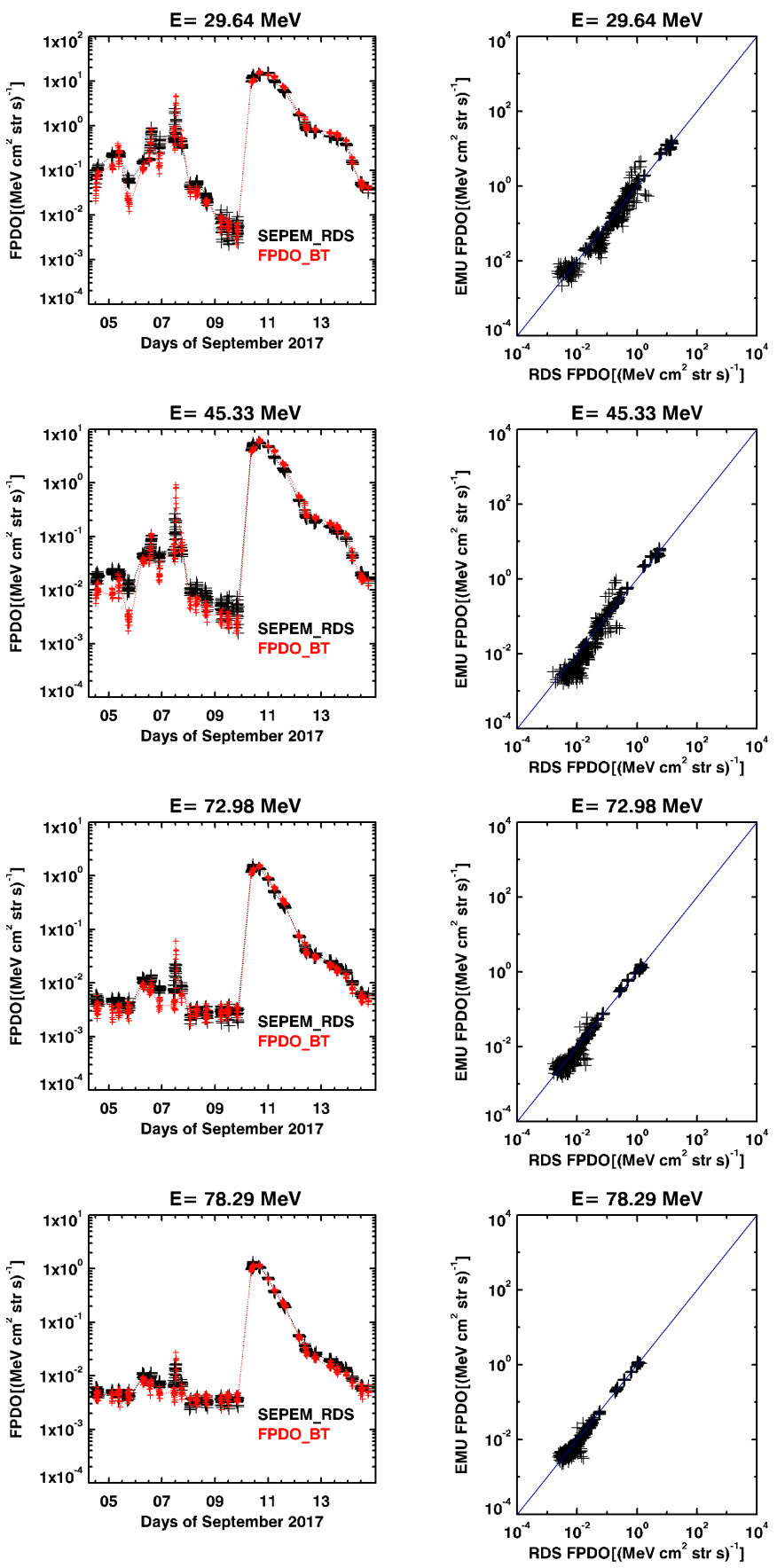

Fig. 9. Cross-plots (on the right hand side) and time series (on the left hand side) of EMU PT proton flux series (red color) and SEPEM RDS flux series (in black) re-binned at the derived effective energies.

means for the characterization of MEO radiation environment along the Galileo orbit. The Galileo EMU electron flux dataset will be used to validate the specifications of Galileo environment and to update MOBE-DIC [2] radiation environment model. In addition, combined studies of MEO and GEO radiation environment will be performed using the opportunity of analyzing data from identical sensors on-board MEO ESA GSAT0207, GSAT0215 and GEO JMA Himawari8,9 missions. 


\section{REFERENCES}

[1] K. A. Ryden et al., "Observations of Internal Charging Currents in Medium Earth Orbit”, IEEE Transactions on Plasma Science, vol. 36, no. 5, pp. 2473-2481, 2008.

[2] A. Hands, K. Ryden, C. Underwood, D. Rodgers, and H. Evans, "A New Model of Outer Belt Electrons for Dielectric Internal Charging (MOBEDIC)", IEEE Transactions on Nuclear Science, vol. 62, no. 6. pp. 2767$2775,2015$.

[3] S.K. Morley, J.P. Sullivan, M.R. Carver, R.M. Kippen, R. H. W. Freidel, G.D. Reeves and M.G. Henderson, "Energetic particle data from the global positioning system constellation", Space Weather, vol. 15, pp. 283-289, $001604,2017$.

[4] S. Bourdarie, D. Lazaro, A. Hands, K. Ryden and P. Nieminen "Electron environment specification models for navigation orbits", in Proceedings of the European Conference on Radiation and its Effects on Components and Systems, pp. 364-368, 5994677, 2009.

[5] A. Sicard-Piet, S. Bourdarie, D. Boscher, R. Friedel, T. Cayton, "Solar cycle electron environment at GNSS like altitudes", in Proceedings of the 57th International Astronautical Congress, International Astronautical Congress (IAF), IAC-06-D5.2.04, 2006.

[6] Johnston, W. R., T. P. OBrien, S. L. Huston, T. B. Guild, and G. P. Ginet , "Recent Updates to the AE9/AP9/SPM Radiation Belt and Space Plasma Specification Model", IEEE Transactions on Nuclear Science, vol. 62, no. 6. pp. 2760-2766, 2015.

[7] K. A. Ryden, A. Hands, C. I. Underwood, and D. J. Rodgers, "Internal Charging Measurements in Medium Earth Orbit Using the SURF Sensor", IEEE Trans. Plasma Science, vol. 43, no. 9, pp. 3014 - 3020, 2015.

[8] H. D. R. Evans et al., "Results from the ESA SREM monitors and comparison with existing radiation belt models" Advances in Space Research, vol. 42, no. 9, pp. 1527-1537, 2008.

[9] T. Nagatsuma, K. Sakaguchi, Y. Kubo, P. Belgraver, F. Chastellain, R. Muff, and T. Otomo, "The Space Environment Data Acquisition Monitor on board Himawari-8 as Space Environment Monitoring on Japanese Meridian of GEO", Earth, Planets and Space, vol. 69, no. 1, p. 75, 2017.

[10] S. Agostinelli et al, "Geant4a simulation toolkit", NIM A, vol. 506, no.3, pp. 250-303, 2003.

[11] G. Santin et al., "GRAS: a general-purpose 3-D Modular Simulation tool for space environment effects analysis", IEEE Transactions on Nuclear Science, vol. 52, no. 6, pp. 2294-2299, 2005.

[12] R. Brun and Rademakers, "ROOT - An object oriented data analysis framework", NIM A, vol. 389, no. 1-2, pp. 81-86, 1997.

[13] J. A. Van Allen, D. N. Baker, B. A. Randall, and D.D. Sentman, "The magnetosphere of Jupiter observed with Pioneer 10, 1. Instrument and principal findings", J. Geophys. Res., vol. 79, no. 25, pp. 3559-3577, 1974.

[14] S. Aminalragia-Giamini, C. Papadimitriou, I. Sandberg et al., "Artificial Intelligence Unfolding for Space Radiation Monitor data", J. Space Weather Space Clim., vol.8, no. 8, A50, 2018.

[15] "SEPEM reference data set (RDS) v2.0 2017a", http://sepem.eu/help/ SEPEM_RDS_v2-00.zip

[16] H. E. Spence et al., "Science Goals and Overview of the Radiation Belt Storm Probes (RBSP) Energetic Particle, Composition, and Thermal Plasma (ECT) Suite on NASAs Van Allen Probes Mission", Space Science Reviews, vol. 179, no.1-4, pp. 311-336, 2013.

[17] J. B. Blake et al., "The Magnetic Electron Ion Spectrometer (MagEIS) Instruments Aboard the Radiation Belt Storm Probes (RBSP) Spacecraft", Space Science Reviews, vol. 4, doi:10.1007/S11214-013-9991-8, 2013.

[18] D.N. Baker et al, "The Relativistic Electron-Proton Telescope (REPT) Instrument on Board the Radiation Belt Storm Probes (RBSP) Spacecraft: Characterization of Earths Radiation Belt High-Energy Particle Populations”, Space Science Reviews, vol. 179, no. 1-4, pp. 383-421, 2012.

[19] UNILIB http://www.mag-unilib.eu/

[20] S. Bourdarie et al., Data analysis procedure, Panel on Radiation Belt Environment Modeling, Available: http://craterre.onecert.fr/prbem/Data_ analysis.pdf

[21] P. Jiggens, C. Clavie, H. Evans et al., "In-Situ Data and Effect Correlation during September 2017 Solar Particle Event", Space Weather, vol. 17, no. 1, pp. 99-117, 2018.

[22] I. Sandberg, P. Jiggens, D. Heynderickx, and I. A. Daglis, "Crosscalibration of NOAA GOES solar proton detectors using corrected NASA IMP-8/GME data", Geophysical Research Letters, vol. 41, no. 3, pp. 44354441, 2014.

\section{ACKNOWLEDGMENT}

We would very much like to thank the engineering team which designed, built and tested the EMUs on Galileo for their excellent work. One of the authors (I.S) is grateful to Dr. Juan V. Rodriguez (UCB CIRES \& NOAA NCEI, USA) and Dr. A. Boudouridis (UCB CIRES \& NOAA NCEI, USA) for inspiring discussions on the applicability of the bow-tie analysis in inverse problems. This work has been supported by ESA/ESTEC contract No. 4000119253/17/NL/LF/hh. The authors acknowledge the National Institute of Information and Communications Technology (Japan) for providing us access to Space Environment Data Acquisition Monitor (SEDA) data. The authors acknowledge Bern Blake, Joe Fennell, Seth Claudepierre and Drew Turner for the use of MagEIS data and Dan Baker, Shri Kanekal, Alyson Jaynes for the use of the REPT data. 